\title{
The road to SuperK-Gd
}

\author{
Lluís Martí Magro* for the Super-Kamiokande Collaboration \\ Kamioka Observatory, Institute for Cosmic Ray Research, University of Tokyo \\ E-mail: martilluesuketto.icrr.u-tokyo.ac.jp
}

From June 2018, the Super-Kamiokande (SK) detector is undergoing a necessary refurbishment to move into its next phase: SuperK-Gd. Dissolving gadolinium (Gd) into the otherwise ultrapure water will add to SK the ability to efficiently tag neutrons. Gd has the largest thermal neutron capture cross-section emitting a gamma cascade with total energy of $8 \mathrm{MeV}$. This has to be compared to the single $2.2 \mathrm{MeV}$ gamma produced from the neutron capture on hydrogen. Neutron tagging capabilities will become a new powerful tool both for signals and background reduction in many analyses.

The test bench for SuperK-Gd has been EGADS, a R\&D project that aimed to demonstrate the feasibility of this idea and that started in 2009. EGADS has shown that Gd sulfate can be easily dissolved and has optimal optical properties to be used in a water Cherenkov detector. At EGADS it has been shown that a very good water quality can be achieved and kept thanks to a novel water purification system specifically designed to keep a good water quality while not removing the dissolved Gd.

XXXIX ICHEP 2018, International Conference on High Energy Physics.

4-11 July 2018

Seoul, Korea

${ }^{*}$ Speaker. 


\section{Introduction}

Super-Kamiokande (SK) has been now running for more than 20 years since in 1996 started operations. The last upgrade was done in 2008 when the data acquisition system was upgraded in order to achieve a high speed signal processing and read out of every hit. The main goals were to be able to cope with the high event rates in case of nearby core-collapse supernovae (SN) and lower energy thresholds. As a consequence, the electron energy threshold for solar neutrino analyses could be lowered to $E_{k i n}=3.5 \mathrm{MeV}$. However, this improvement is not enough to efficiently detect the single $2.2 \mathrm{MeV}$ gamma that follows a neutron capture on hydrogen.

The ability to tag neutrons would open up new possibilities in many of our current analyses: diffuse supernova (SN) neutrino background (DSNB): the neutrinos from all the past core-collapse SN in the universe, improving the pointing accuracy in case of a SN in our galaxy and early warning for nearby SNe are some of the most straightforward applications. For more details see [1]. Another important application is background reduction in proton decay studies since in most of the possible decay channels there is no accompanying neutron.

In order to make efficient neutron tagging possible, GADZOOKS! [2] was proposed in 2004: dilute $0.1 \%$ of Gd in mass into the SK ultra-pure water. Having the largest thermal neutron capture cross-section (49000 barn) and producing a $8 \mathrm{MeV}$ gamma cascade (to compare to the 0.33 barn for hydrogen and the single $2.2 \mathrm{MeV}$ gamma produced when capturing a neutron) makes Gd a perfect candidate to enable neutron tagging. Metallic $\mathrm{Gd}$ does not dissolve but $\mathrm{Gd}$ sulfate dissolves very easy and as will be shown in section 2 the optical properties are optimal for water Cherenkov detectors.

In 2009 the R\&D project EGADS was approved to demonstrate the feasibility of GADZOOKS! For this purpose five goals were set:

- Show that the filtration system can achieve and maintain a good water quality while keeping the Gd concentration in water constant.

- Show that Gd sulfate has no adverse effects on detector components.

- Show that it can be added/removed in an efficient and economical way.

- Show that other analyses will not be affected.

- Show that the now visible neutron background, and specially that from impurities in the $\mathrm{Gd}$ sulfate itself, does not represent a problem.

In addition to the above, the existing water leak in the SK tank had to be repaired in order to avoid releasing Gd into the environment.

\section{EGADS}

EGADS is a 200-ton tank with 200 photosensors and its own water purification system which was specifically designed to meet the above criteria. The water was monitored in three positions: 
top, centre and bottom. The Cherenkov light left after travelling $15 \mathrm{~m}$ (LL15), with PMT efficiencies included is plotted in Fig. 1. The blue band represents the typical LL15 values for SKIII/SK-IV phases. The Gd sulfate concentrations are also monitored at the same positions, where the horizontal dashed line represents the Gd sulfate target concentration. After every Gd sulfate loading (vertical dashed areas) we observe a momentarily LL15 decrease in all positions but then the filtration system recovers the water quality back into SK typical values. In fact, whenever the running conditions are stable the LL15 values are within the blue band. The Gd sulfate concentration increases after each loading in all positions. The concentration shows a good uniformity in all positions. In addition, we do not observe any losses since the last loading (end of April 2015) until the end of the run (October 2017). Thus, for about 2.5 years no losses were observed.

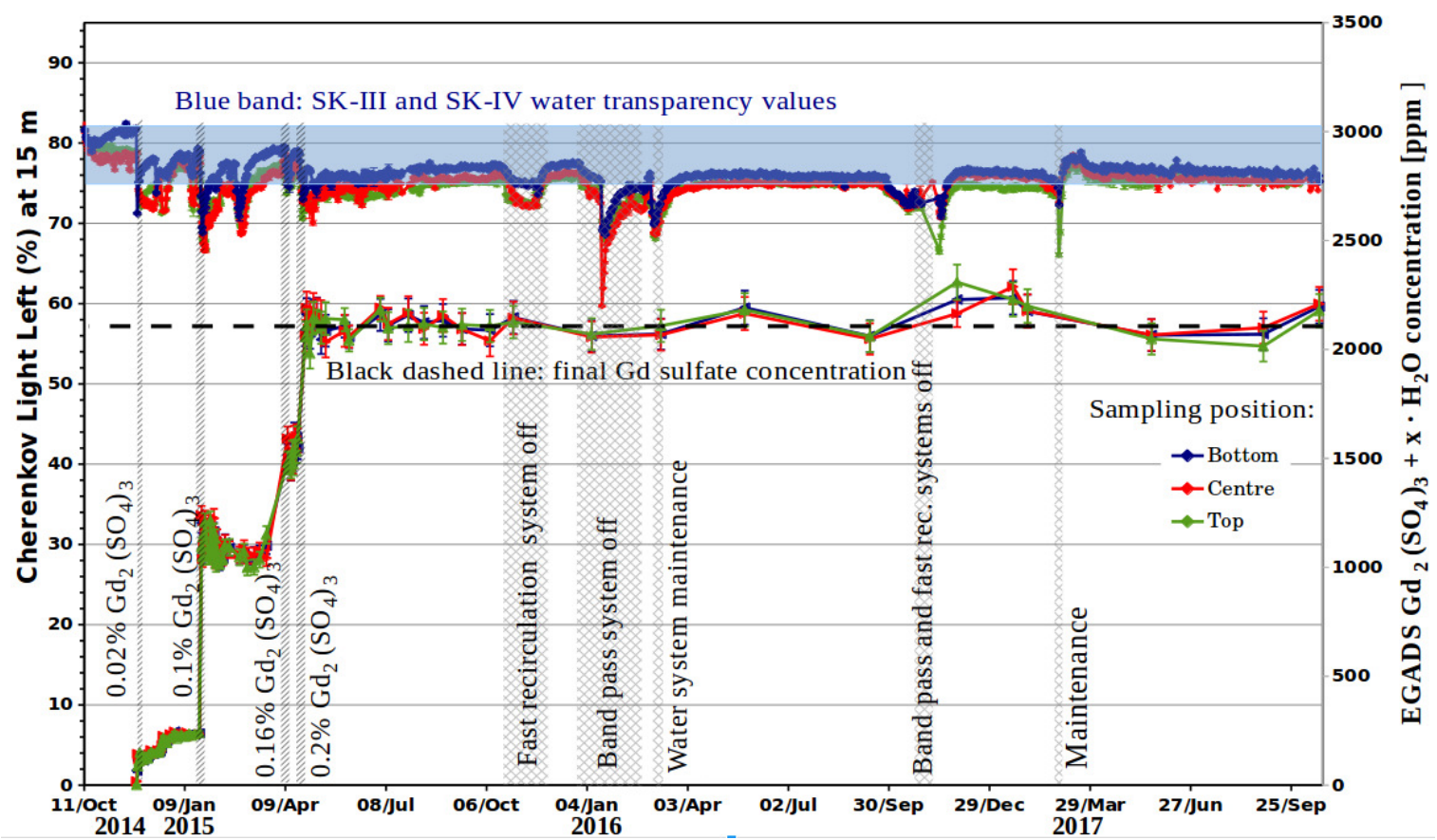

Figure 1: Cherenkov light left [\%] at $15 \mathrm{~m}$ and Gd sulfate concentration for the three sampling positions in the EGADS detector: bottom, centre and top (blue, red and green, respectively).

After that, EGADS was drained. A special resin that removes Gd was used in order to avoid releasing Gd into the environment. This procedure had been tested previously. We could not find any trace of Gd using an ICP/MS and we concluded that the resulting concentration was $<0.5 \mathrm{ppb}$.

Once the tank was empty we entered inside to perform an eye inspection of the structure, photosensors and other elements. We found no evidence of ageing or damage.

We measured the typical impurities of Gd sulfate batches we had been using so far, see table 1 . We also conducted studies to determine the radio-purities requirements for our analyses. These impurities can mimic their signals and thus, they need to be set under control. If no number is given (-) it means that the corresponding requirement is less restrictive.

We have collaborated with several companies to produce a Gd sulfate powder that meets our requirements. They produce highly radiopure Gd sulfate, give us those baches and we test the radiopurity with low background germanium detectors at Canfranc (Spain) and by ICP/MS mea- 


\begin{tabular}{c|c|c|cc} 
Chain & $\begin{array}{c}\text { Part of the } \\
\text { chain }\end{array}$ & $\begin{array}{c}\text { Typical } \\
(\mathrm{mBq} / \mathrm{Kg})\end{array}$ & $\begin{array}{c}\text { DSNB } \\
(\mathrm{mBq} / \mathrm{Kg})\end{array}$ & $\begin{array}{c}\text { Solar } \\
(\mathrm{mBq} / \mathrm{Kg})\end{array}$ \\
\hline${ }^{238} \mathrm{U}$ & ${ }^{238} \mathrm{U}$ & 50 & $<5$ & - \\
& ${ }^{226} \mathrm{Ra}$ & 5 & - & $<0.5$ \\
\hline${ }^{232} \mathrm{Th}$ & ${ }^{232} \mathrm{Th}$ & 10 & - & $<0.05$ \\
& ${ }^{228} \mathrm{Th}$ & 100 & - & $<0.05$ \\
\hline${ }^{235} \mathrm{U}$ & ${ }^{235} \mathrm{U}$ & 32 & - & $<3$ \\
& ${ }^{227} \mathrm{Ac} /{ }^{227} \mathrm{Th}$ & 300 & - & $<3$ \\
\hline \hline
\end{tabular}

Table 1: Relevant radioactive contamination and typical impurities in untreated Gd sulfate and our requirements from our DSNB and solar neutrino physics goals.

surements at Kamioka (Japan) [3]. Within experimental uncertainties, one of these companies already meets our requirements while others are still working to achieve them.

\section{SK detector refurbishment}

To be able to stop the leak at SK, we chose a two layer strategy with two different sealants and two different mechanical properties: BIO-SEAL 197 by Thin Film Technology Inc. and MineGuard-C by Hodogawa company. These sealants have been applied in all potential leak locations like weld seams between stainless steel plates, PMT support structures, etc. While BIO-SEAL 197 can easily sneak in cracks and is mechanically very strong although rigid, MineGuard-C, which overcoats the BIO-SEAL 197 film, is more flexible, allows for larger displacements and has low Rn emanation levels. Many tests were conducted with these two materials to ensure they meet our mechanical and contamination requirements.

EGADS showed very good results and thus, in June 2015 the SuperK-Gd project was approved. From June 2018 the refurbishment work started and it is expected to finish by January 2019. In addition to the water leak fix work, PMTs in the inner (ID) and outer (OD) detectors were replaced: about 140 and 200, respectively. The Tyvek sheets that optically separate the ID and OD were also replaced.

Early next year expect to resume the new physics phase with pure-water. The dates for $\mathrm{Gd}$ loading will then be decided once we have assessed the leak has been fixed and in coordination with the T2K collaboration.

\section{References}

[1] SUPER-KAMIOKANDE collaboration, Charles Simpson, Physics Potential of SuperK-Gd.

[2] J. F. Beacom and M. R. Vagins, "GADZOOKS! Anti-neutrino spectroscopy with large water Cherenkov detectors,” Phys. Rev. Lett. 93, 171101 (2004) doi:10.1103/PhysRevLett.93.171101 [hep-ph/0309300].

[3] S. Ito, Y. Takaku, Y. Kishimoto and M. Ikeda, "Determination of Trace Levels of Uranium and Thorium in High Purity Gadolinium Sulfate Using ICP-MS with Solid-Phase Chromatographic Extraction Resin,” arXiv:1709.03417 [physics.ins-det]. 